\title{
Corrigendum
}

Corrigendum: Correction of Acknowledgments

\section{Corrigendum: We Take Care of Person with Obesity, Not Obesity That People Has}

\section{Sang Yeoup Lee*}

Family Medicine Clinic and Research Institute of Convergence of Biomedical Science and Technology, Pusan National University Yangsan Hospital, Yangsan; Department of Medical Education, Pusan National University School of Medicine, Yangsan, Korea

J Obes Metab Syndr 2019;28:73-75

https://doi.org/10.7570/jomes.2019.28.2.73

In my recently published article, the statement for a funding source was missing. It should be properly added as follows. We would like to apologize for any inconvenience or misunderstanding.

\section{ACKNOWLEDGMENTS}

This work was supported by a 2-year research grant of Pusan National University. 